\title{
Quantitative study of the development of isolated mouse pre-antral follicles in collagen gel culture
}

\author{
C. Torrance*, Evelyn Telfer and R. G. Gosden \\ Department of Physiology, Medical School, University of Edinburgh, Teviot Place, \\ Edinburgh EH8 $9 A G, U K$
}

\begin{abstract}
Summary. Follicles were isolated from the ovaries of 10 -day-old C57BL6/CBA $F_{1}$ hybrids by mechanical and enzymic treatment, embedded in a collagen-gel matrix to maintain the 3 dimensional integrity of the follicle and cultured for up to 14 days. Gels were removed at various times during the culture period and prepared for histology. Follicles grew from unilaminar to multilaminar stages within 6 days of the culture period. A more detailed assessment of growth by counting follicles at different stages and measuring oocyte and follicle diameters showed that follicle growth was maintained for up to 14 days in culture. Initially the proportion of unhealthy follicles was high but this declined after 6 days in culture.
\end{abstract}

Keywords: pre-antral follicles; collagen gel; culture; mouse

\section{Introduction}

The follicle is the functional unit of the mammalian ovary and has a dual role-it provides the micro-environment for oocyte growth and maturation and is responsible for sex steroid production. The ovary of an adult mouse contains thousands of follicles at various stages of development. There are three basic types of follicle: (1) non-growing or 'primordial' follicles, (2) preantral follicles in which oocyte growth and increase in granulosa cell numbers and layers is occurring, and (3) antral follicles which contain a fully grown oocyte with granulosa cells and a layer of differentiated theca cells. Primordial follicles move continually from the non-growing pool to enter a growth phase.

Follicles progress towards the antral stage without a further resting stage: a few are ovulated, the majority of follicles are lost through atresia. Little is known of the factors initiating and controlling growth of small follicles and extrapolation from the detailed knowledge of antral follicles may be unreliable, although a useful starting point. Recently, attention has concentrated on the role of paracrine and autocrine mechanisms in controlling ovarian function (reviewed by Hsueh, 1986). Various studies, mostly conducted in vitro using monolayers of granulosa cells, have indicated effects on growth and maturation of ovarian steroids (androgens, oestrogens and progesterone), and peptides (GnRH-like peptides, EGF, TGF- $\beta$, insulin, insulin-like growth factor-I (IGF-I), vasoactive intestinal peptide (VIP)) and even neurotransmitter substances. Most of these studies involved granulosa monolayers as the model, yet it is not clear how closely the state of differentiation reflects that in vivo. Evidence has been obtained that the secretory activity of granulosa cells is altered by cytoskeletal inhibitors and by culture in collagen gel, both of which are expected to affect the shape of the cell (Carnegie \& Tsang, 1988).

To mimic physiological conditions it is preferable to study follicle growth and metabolism in isolated intact follicles in which normal three-dimensional relationships are maintained between

*Present address: Department of Nursing Studies, College of Medicine, University of Wales, Heath Park, Cardiff CF4 4XN, UK. 
granulosa cells and the oocyte. Furthermore, separation of the granulosa-oocyte unit from the stroma/theca cells at the basement membrane interface is a potentially valuable method for investigating cellular interactions. In this study a technique has been developed to isolate and culture intact follicles in a collagen-gel matrix.

\section{Materials and Methods}

Animals. The mice used in this study were $\mathrm{F}_{1}$ females aged 8-11 days post partum and derived from matings between $\mathrm{CBA} / \mathrm{Ca}$ males and $\mathrm{C} 57 \mathrm{BL} / 6$ females.

Culture media. All preparatory steps were carried out in Hepes-buffered medium M199 (Gibco, Paisley, UK) and follicles were cultured in bicarbonate-buffered M199 (Gibco). Both media were supplemented with gentamicin (Sigma, Poole, Dorset, UK), amphotericin B (Sigma), L-glutamine (Flow Laboratories, Irvine, UK) and sodium pyruvate (BDH, Poole, Dorset, UK). The culture media also contained 10\% donor calf serum (Flow Laboratories) which was found by bioassay to contain undetectable amounts of gonadotrophins (C. Torrance, unpublished). Sera were heat-inactivated at $55^{\circ} \mathrm{C}$ for $35 \mathrm{~min}$. Preparation media had an osmolality of $290-310$ mosmol kg ${ }^{-1}$ as measured by a Wescor vapour pressure osmometer and a $\mathrm{pH}$ of $7 \cdot 4$. The culture media had an osmolality within a range of 280-300 mosmol kg-1.

Collagen-gel solution. Collagen gel was extracted from the tail tendons of rats by the methods of Ehrmann \& Gey (1956) and Chambard et al. (1981). The tendons were transferred to $70 \%$ alcohol, and rinsed in sterile distilled water. Then $1 \mathrm{~g}$ of tendon was added to $100 \mathrm{ml}$ 1:1000 acetic acid and stirred at $4^{\circ} \mathrm{C}$ for $48 \mathrm{~h}$. The solution was centrifuged at $2000 \mathrm{~g}$ on a benchtop centrifuge for $1 \mathrm{~h}$. The solution was stored at $4^{\circ} \mathrm{C}$ for up to 8 weeks. Gels were prepared immediately before use by mixing $200 \mu \mathrm{l}$ serum with $200 \mu$ l concentrated $(\times 10)$ Medium 199 at $4^{\circ} \mathrm{C}$. The $\mathrm{pH}$ was adjusted by adding $500 \mathrm{~mm}-\mathrm{NaOH}$ until the indicator (phenol red) turned the appropriate colour for physiological $\mathrm{pH}$.

Follicle isolation. All steps were carried out using cold preparation media and in each experiment 24 mice were used. The animals were killed by decapitation and the ovaries were removed and placed in a watchglass containing medium. All subsequent steps were carried out using sterile techniques in a laminar flow hood. The ovaries were bisected with fine needles. The bisected ovaries were incubated with gentle rotation at $37^{\circ} \mathrm{C}$ in a $10 \mathrm{ml}$ tube containing medium with $1.5 \mathrm{mg}$ collagenase $/ \mathrm{ml}$ (Sigma, Type 1) and $40 \mathrm{U}$ DNAse $\mathrm{I} / \mathrm{ml}$ (Sigma). After $30 \mathrm{~min}$ the ovaries were centrifuged $(100-200 \mathrm{~g})$ the tissue and the supernatant was removed. The pellet was resuspended in $10 \mathrm{ml}$ medium and centrifugation repeated. The follicles were resuspended in $3 \mathrm{ml}$ medium and transferred to a watchglass.

Under a dissecting microscope follicles were isolated by repeated pipetting of the bisected ovaries using Gilson pipettes. As the fragments decreased in size pipette tips of progressively smaller diameter were used. To minimize mechanical trauma from repeated pipetting, freed follicles were harvested frequently and filtered through a $125 \mu \mathrm{m}$ nylon mesh (Nybolt ASTM 120-125, Simon Ltd, Stockport, Cheshire, UK) to remove lumps. The filter was flushed to remove trapped follicles. The follicles were pelleted by gentle centrifugation, the liquid was removed and the follicle pellet was resuspended in $3 \mathrm{ml}$ medium.

The pellet was transferred to a watchglass and gently pipetted to break up clumps. Gentle rotation concentrated the follicles in the centre of the watchglass. Then $20 \mu \mathrm{l}$ samples were pipetted from the central concentration into the wells of a Terasaki plate (Flow Laboratories). The rotation was repeated until 16 samples had been obtained. A Kleenex tissue was used to remove excess fluid from the wells and $10 \mu l$ of the collagen-gel solution were added and pipetted once to suspend the follicles. The follicles were incubated for $2-3 \mathrm{~min}$ at $37^{\circ} \mathrm{C}$ until the gel had set. After $20 \mu \mathrm{l}$ gel solution were pipetted into empty wells, the $10 \mu \mathrm{l}$ set gels were transferred to them. The double gels were allowed to set as before. Double gelling was necessary to avoid follicle losses resulting from collagen gel contraction during culture and processing for histology. Four gels were transferred to the wells of a Linbro tissue-culture plate containing $2 \mathrm{ml}$ culture medium. The gels were incubated at $37^{\circ} \mathrm{C}$ with $5 \% \mathrm{CO}_{2}$ in a humidified incubator. The medium was changed $24 \mathrm{~h}$ later and every 3 rd day.

Follicles could be monitored during culture by using inverted phase-contrast microscopy because collagen gel is transparent. At the termination of culture the gels were fixed overnight in aqueous Bouin's fluid, embedded in paraffin wax and sectioned at $5 \mu \mathrm{m}$. Sections were stained with haematoxylin and eosin and mounted with DPX.

Classification of follicles. Follicles were observed under $\times 400$ magnification and classified according to the number of cell layers surrounding the oocyte, based on the established system of Mandl \& Zuckerman (1951): Stage I, oocyte surrounded by a single layer of squamous granulosa cells; Stage II, growing oocyte surrounded by a single layer of cuboidal granulosa cells; Stage III, growing oocyte surrounded by 2 layers of cuboidal granulosa cells; Stage IV, growing oocyte surrounded by 3 layers of cuboidal granulosa cells; Stage V, oocyte surrounded by 4 or more layers of cuboidal granulosa cells but no antrum.

Follicles were also classed as disrupted (including free oocytes and any follicles with a disrupted granulosa layer) or intact (granulosa layer(s) intact). The quality of the intact follicles was also assessed. Follicles were classed as intact but damaged if they had more than slight contraction of the oocyte from the granulosa layers, with nuclear contraction, with obvious separation of the granulosa layers, or with more pycnotic cells than cell layers. 
Longitudinal study of follicle growth. Experiments were designed to establish the pattern of follicle growth in collagen-gel culture. For each experiment 4 gels from one well were allowed to equilibrate in the incubator for $1 \mathrm{~h}$ and then harvested for histology to obtain information on the numbers, distribution and quality of follicles in the gels at the beginning of the culture period $(0 \mathrm{~h})$. Groups of 4 gels from the same well were harvested at intervals during the culture period (see Fig. 1) so that an impression of the pattern of growth over a 14-day culture period would be gained. The results from 11 disaggregation experiments and more than 100 gels are reported. Differential follicle counts using the above classification system were carried out on all gels.

Every section was counted in the 0 -h gels and in gels cultured for 6 or more days in which numbers were lower. Every 3rd section was counted for gels from 1 to 5 days in culture. Follicles were counted only when the follicle section contained the nucleolus of the oocyte. Nucleolar diameters were measured in 22 follicles for Stage I, II and III follicles in 0-h gels using an image-shearing micromeasurement system (Vickers Instruments, York, UK) under $\times 100$ oil immersion optics. These measurements were used to estimate a correction factor to compensate for overcounting resulting from the possibility of the nucleolar marker being present in more than one section. Total numbers of follicles were calculated by multiplying the number counted by 3 (the sampling frequency) and the correction factor. As Stage V follicles were absent in the 0-h gels, the Stage IV correction factor was used. Stage I (primordial) follicles were present in the gels but were not counted.

\section{Results}

Figure 1 summarizes the data of the follicle counts for individual gels over the course of the culture. The mean number of follicles declined with time. The proportion of follicles intact but showing some histological evidence of damage ranged from 33 to $66 \%$ between Days 2 and 5. On Days 6-8 the percentages of these follicles decreased to 22,28 and $17 \%$, and increased after Day 9 to $40 \%$, reaching $64 \%$ by Day 14 .

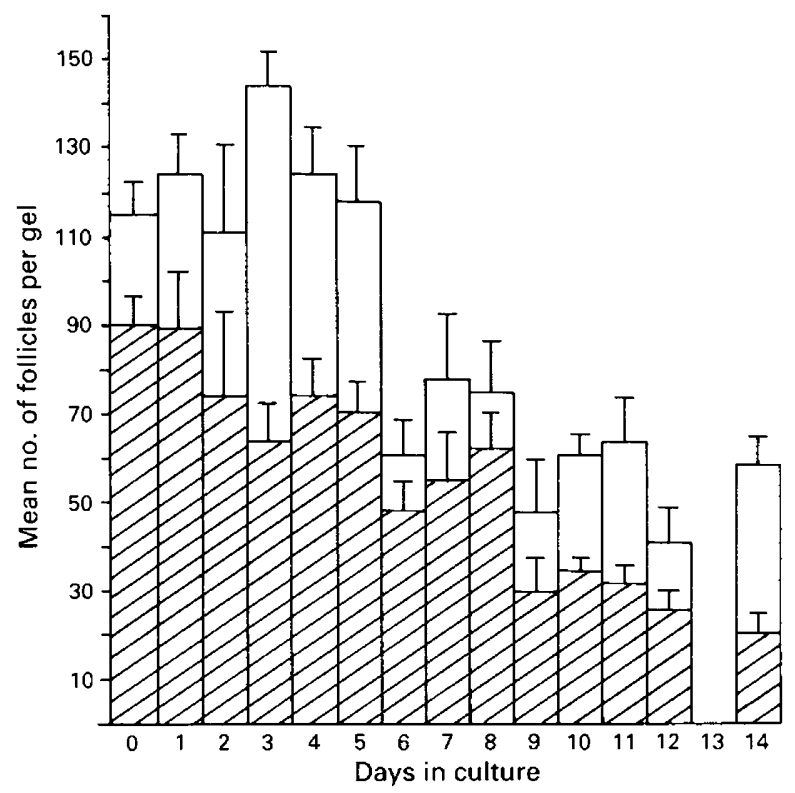

Fig. 1. A representation of the mean total number of follicles (all stages) per gel over 14 days in culture. The hatched bars represent morphologically normal follicles and the unhatched bars represent disrupted follicles.

The distribution of follicle stages underwent a marked shift with time, with small stages gradually disappearing and large ones emerging. Figure 2 shows the distribution of follicles of each stage over the course of 14 days in culture. At the start of the culture period $(0 \mathrm{~h}) 43 \%$ of follicles were Stage II and $42 \%$ Stage III with only $5 \%$ being Stage IV. After a 6-day culture period the 
distribution of follicle stages had altered with $36 \%$ of follicles being Stage I1, 30\% Stage III, $17 \%$ Stage IV and $10 \%$ Stage V (i.e. with 4 or more layers). After 14 days in culture this distribution had shifted to only $16 \%$ of follicles being Stage II, 16\% Stage III, $24 \%$ Stage IV and $44 \%$ of follicles being Stage V. Stage V follicles contained 4 or more layers yet remained preantral. Antral follicles were not identified at any time and occasional spaces between granulosa cells were attributed to histological artefacts.

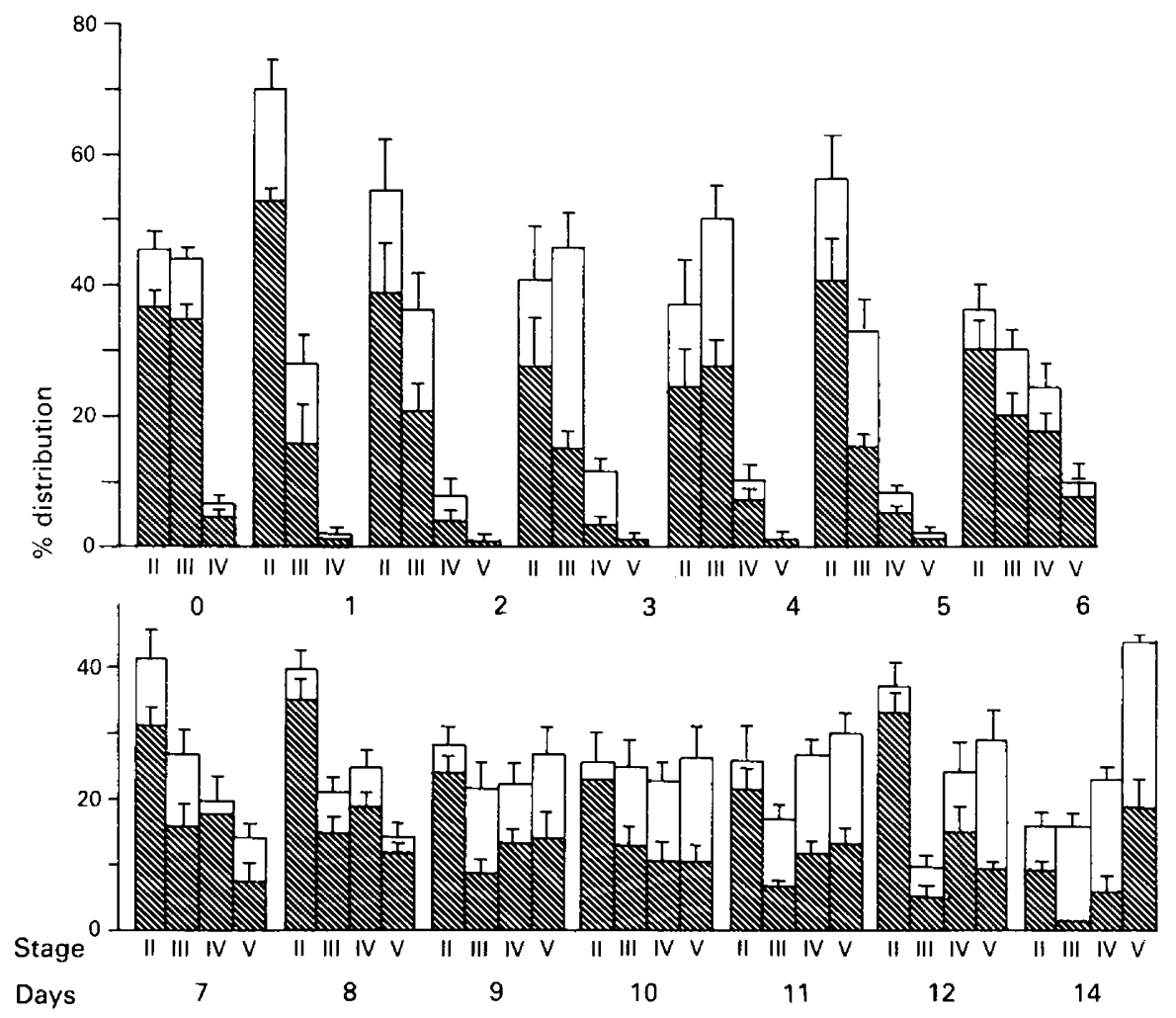

Fig. 2. Distribution of follicle stages (II-V) as a percentage of the total number of intact follicles per gel from 0 to 14 days in culture. Hatched area represents the $\%$ of intact follicles with no histological evidence of degeneration. Values are given as mean \pm s.e.m.

Between Days 6 and 8 of culture a reduction in disruption of follicle stages was found. From the start of the culture period up to Day 5 there was a gradual increase in the proportion of larger follicle types but the proportion of unhealthy follicles was high. From Day 6 the proportion of unhealthy follicles dropped and by Day 8 of the culture period $80 \%$ of follicles at all stages were histologically normal. By Day 14, however, fewer than half the follicles at any given stage were healthy.

Observation of the gels by phase-contrast microscopy during culture indicated that the follicles retained their spheroidal form, with the oocyte maintaining a central position (Fig. 3). Numerous processes were observed growing outwards from the granulosa cells. As the cultures progressed the collagen gels tended to shrink, making viewing more difficult. After several days in collagen gel culture follicles maintained an appearance (Fig. 4) indistinguishable from that in the intact ovary (Fig. 5). The major difference between the intact ovary and the cultured follicles was 


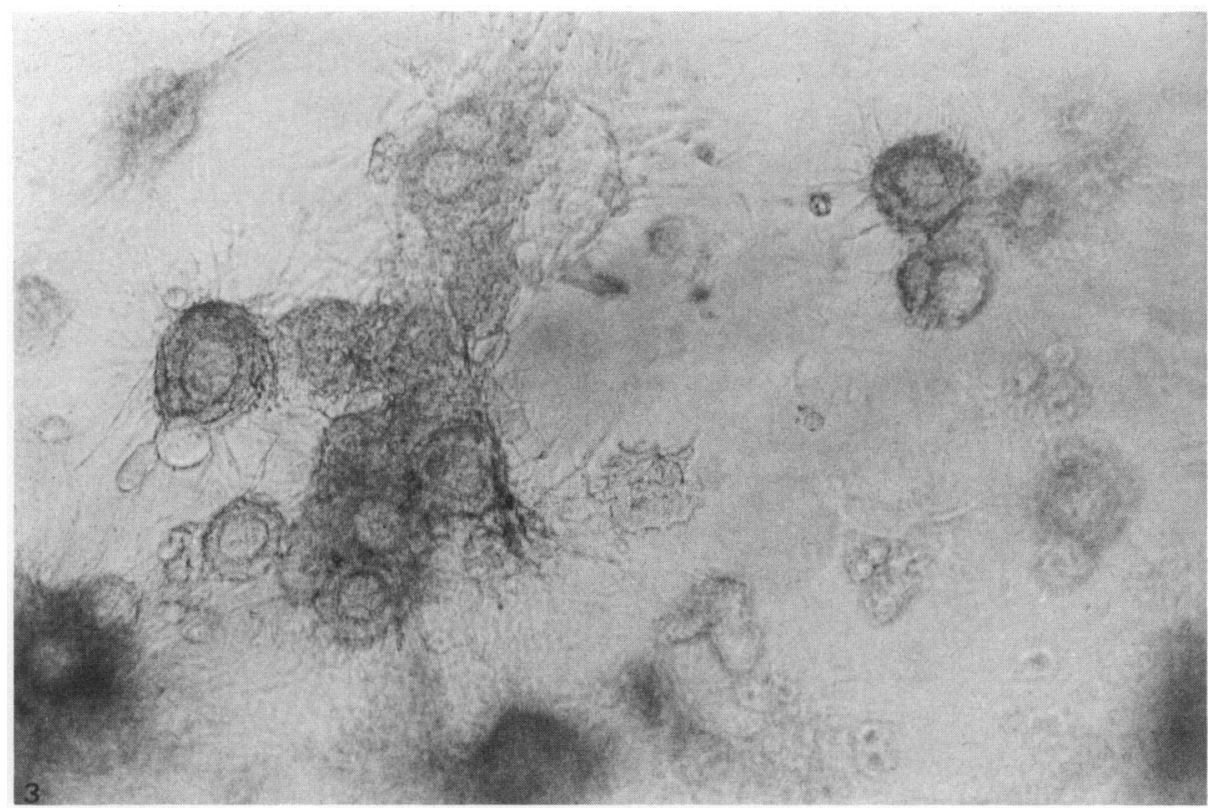

Fig. 3. Follicles in collagen gel during the culture period. The follicles maintain their structure and show cellular outgrowths. $\times 150$.

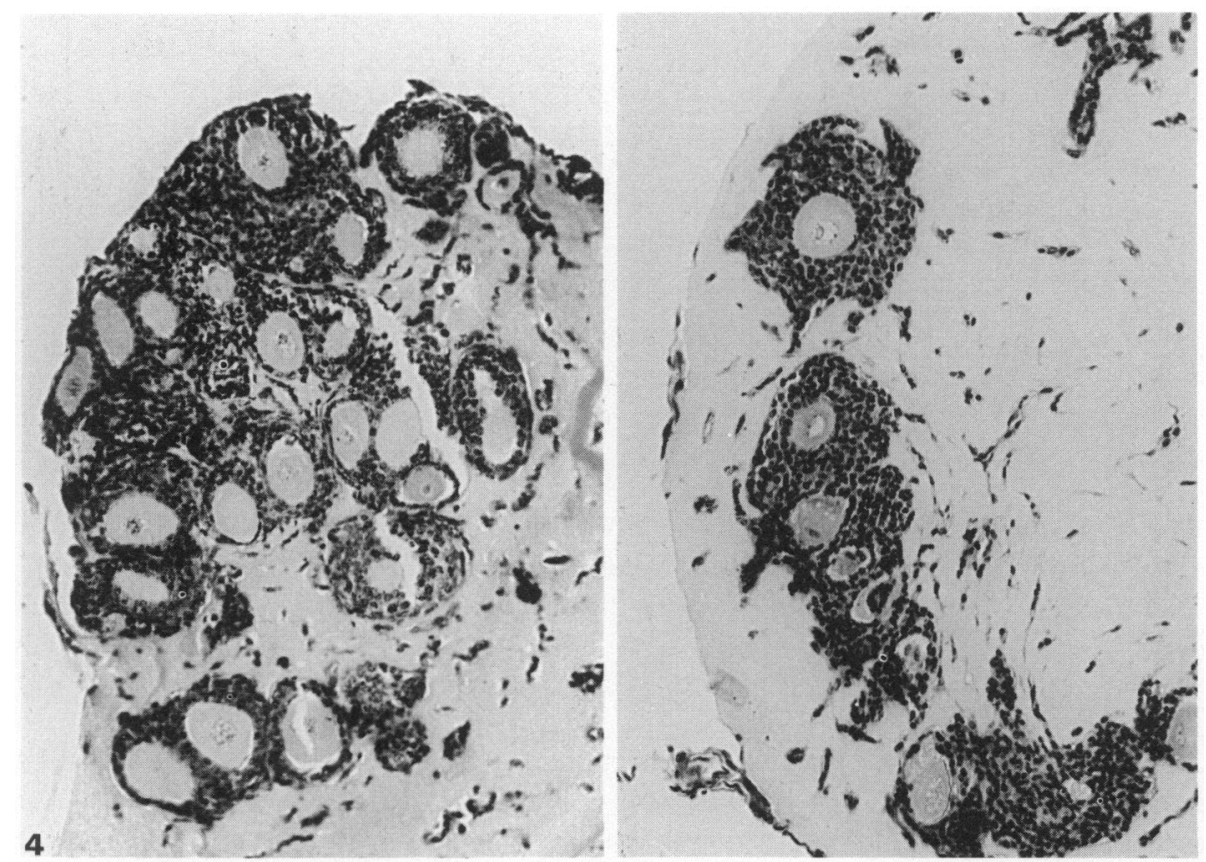

Fig. 4. Histological sections of a gel containing follicles after 6 days in culture. Unilaminar and multilaminar follicles can be seen. Some of the multilaminar follicles are showing signs of degeneration. $\mathrm{H} \& \mathrm{E}, \times 150$. 


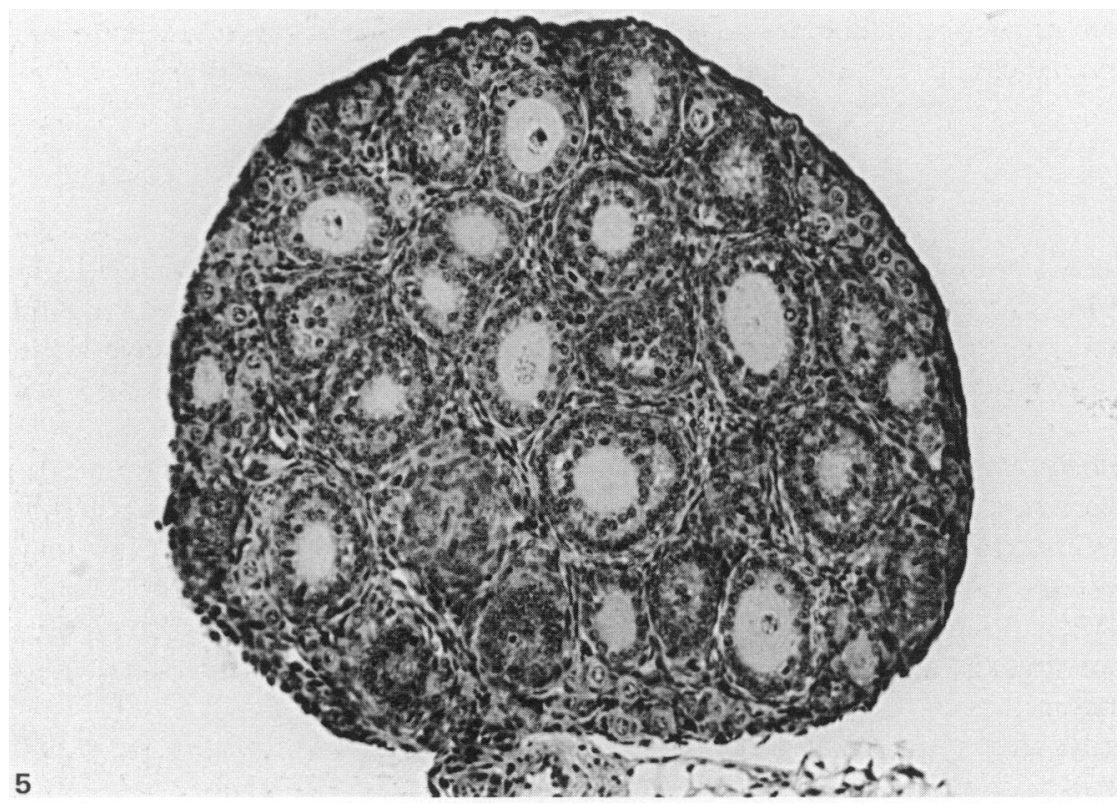

Fig. 5. Section of an intact ovary from a 10 -day-old $\mathbf{F}_{1}$ hybrid mouse. No multilaminar follicles are found in ovaries at this age. $\times 150$.

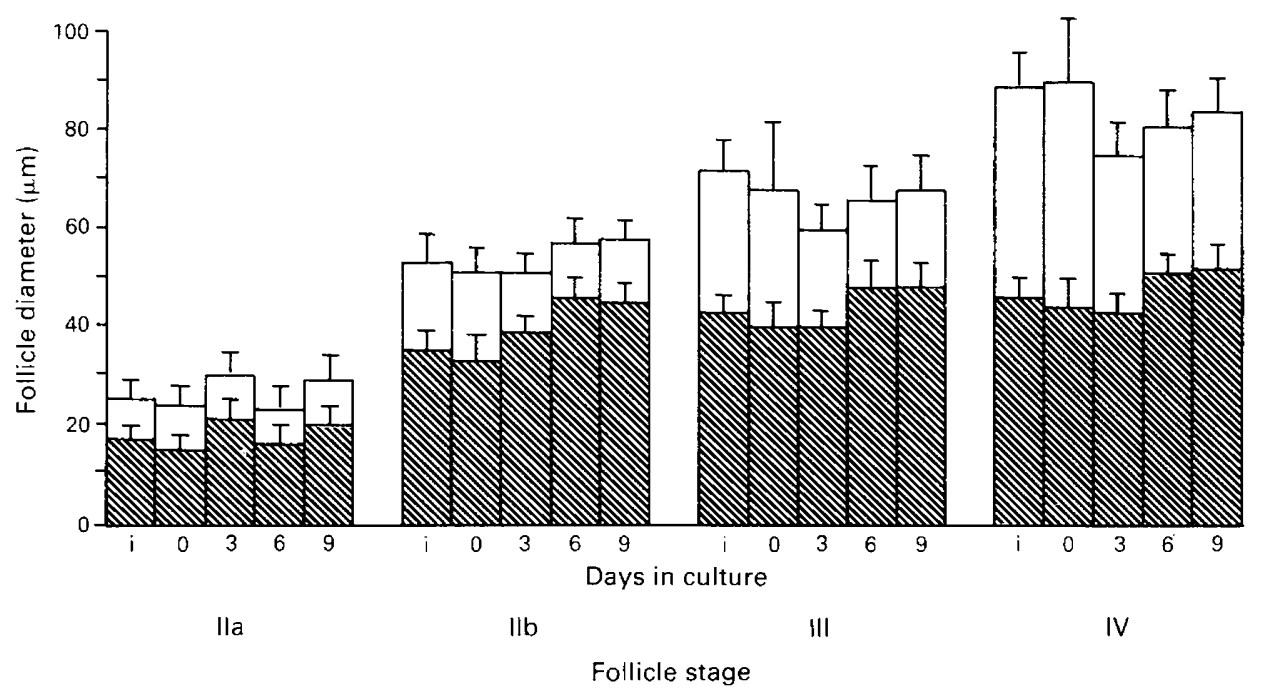

Fig. 6. Mean follicle and oocyte (hatched area) diameters at four follicle stages in intact ovaries (i) and isolated follicles up to 9 days in culture. Values are given as mean \pm s.d. for at least 50 follicles.

the presence of multilayered follicles after 6 days in culture. Stage- $V$ follicles were absent from the 10-day-old ovary (Fig. 5) but represented $27 \%$ of the intact follicles by Day 12 in culture. Although no counts were made of primordial follicles, they were present at all times and were histologically normal. 
The oocyte and follicle increased in size at each stage in culture in all groups (Fig. 6). There were no significant differences in oocyte and follicle diameter with time in the culture compared with the intact ovary, indicating that follicular development was proceeding normally.

\section{Discussion}

A technique has been developed which permits mouse ovarian follicles to be isolated and grown in vitro for at least 2 weeks. Using this dissociation procedure large numbers of isolated follicles can be produced. The 10-day-old mice were chosen because they do not contain Stage V-follicles (follicles with 4 or more layers). The multilaminar follicles seen after 6 days in culture have therefore emerged in vitro from small or even primordial follicles. Roy \& Greenwald (1985) developed a similar dissociation method using collagenase and pronase to isolate the follicles of hamsters but have only carried out short-term incubations to study hormone production and receptors (Roy et al., 1987). Without a gel matrix the follicular unit is disrupted but in collagen gel apparently normal growth proceeds without evidence of limiting diffusion of gases and metabolites. The collagen-gel matrix provides other advantages, i.e. a convenient method for handling follicles during histological processing and a convenient vehicle for transplanting follicles to the kidney capsule where Graafian maturation can occur (Torrance et al., 1988).

These advantages of collagen gels have been exploited with several cell types, e.g. mammary epithelial cells (Yang \& Nandi 1983) and thyroid epithelial cells (Chambard et al., 1981). There is evidence that even with granulosa cell monolayers the purine hypoxanthine can maintain oocytegranulosa cell complexes isolated from 10-11-day-old mice (Eppig \& Downs, 1987). It would seem that hypoxanthine is maintaining functional relationships between the oocyte and granulosa cells because some oocytes are competent to be fertilized after culture but the effects of hypoxanthine on follicle units in collagen-gel culture have yet to be tested.

The culture system has been shown to be capable of supporting apparently normal follicle development up to multilaminar stages. These follicles are clearly growing from unilaminar follicles, but whether primordial follicles are beginning to grow in this system cannot as yet be determined. The relatively high proportion of unhealthy follicles present during the first few days in culture suggests that this is as a result of damaged follicles being put into culture after the dissociation. The reduction in the proportion of unhealthy follicles at all stages after 6 days in culture supports the view that follicle growth is proceeding normally. While intact small follicles often appeared identical to their in-vivo counterparts many of the multilaminar follicles showed signs of degeneration and the development of an antrum has not been observed in any of the cultured follicles. It would appear that the multilaminar follicles in culture had reached a stage where they might require additional support to continue growing. Media supplemented with donor calf serum were chosen to represent basal conditions as this serum contains little gonadotrophin or sex steroids but can be expected to contain other growth factors. These media may not be appropriate to support growth in later stages and we are now trying to characterize these growth requirements to obtain a chemically defined medium capable of supporting all stages of preantral follicle growth.

The gels do not only contain intact follicle units but also a variety of cells, including granulosa cells, stromal cells, ovarian surface epithelium, and other cell types. Since it would be useful to separate follicles into stages to study stage-specific effects or secretions, a method of producing a purified follicle suspension is being sought. The separation of follicles on a small scale has been achieved by using calibrated pipettes (Roy \& Greenwald, 1985) but a method which would allow large numbers to be conveniently handled is required.

We thank Kay Grant for technical assistance with the histology; The Faculty of Medicine, University of Edinburgh for a scholarship to C.T.; and The Wellcome Trust for a grant to R.G.G. 


\section{References}

Carnegie, J.A. \& Tsang, B.K. (1988) The cytoskeleton and rat granulosa cell steroidogenesis: possible involvement of microtubules and microfilaments. Biol. Reprod. 38, 100-108.

Chambard, M., Gabrion, J. \& Mauchamp, J. (1981) Influence of collagen gel on the orientation of epithelial cell polarity: follicle formation from isolated thyroid cells and from preformed monolayers. $J$. Cell Biol. 91, 157-166.

Ehrmann, R.L. \& Gey, G.O. (1956) The growth of cells on a transparent gel of reconstituted rat-tail collagen. J. natn. Cancer Inst. 16, 1375-1403.

Eppig, J.J. \& Downs, S.M. (1987) The effect of hypoxanthine on mouse oocyte growth and development in vitro: Maintenance of meiotic arrest and gonadotropin-induced oocyte maturation. Devl Biol. 119, 313-321.

Hsueh, A.J.W. (1986) Paracrine mechanisms involved in granulosa cell differentiation. Clin. in Endocr. Metabol. 15, 117-134.
Mandl, A.M. \& Zuckerman, S. (1951) The relation of age to numbers of oocytes. J. Endocr. 7, 190-193.

Roy, S.K. \& Greenwald, G.S. (1985) An enzymatic method for dissociation of intact follicles from the hamster ovary: histological and quantitative aspects. Biol. Reprod. 32, 203-215.

Roy, S.K., Wang, S.C. \& Greenwald, G.S. (1987) Radioreceptor and autoradiographic analysis of FSH, hCG and prolactin binding sites in primary to antral hamster follicles during the periovulatory period. $J$. Reprod. Fert. 79, 307-313.

Torrance, C., Telfer, E. \& Gosden, R.G. (1988) An investigation of mouse ovarian follicles in collagen gel culture. J. Reprod. Fert., Abstr. Series 1, p. 24, abstr.

Yang, J. \& Nandi, S. (1983) Growth of cultured cells using collagen as a substrate. Int. Rev. Cytol. 81, $249-286$.

Received 14 February 1989 